\title{
U-BeComp: Plataforma de Composición de Servicios para Ambientes de Computación Ubicua
}

\section{U-BeComp: Service Composition Platform for Ubiquitous Computing Environments}

\begin{abstract}
Resumo: La computación ubicua se ha establecido como la siguiente generación de las comunicaciones, ofreciendo a los usuarios las capacidades de la redes en cualquier momento y lugar, con el fin de soportar sus tareas cotidianas. Sin embargo, el despliegue de servicios en ambientes ubicuos requiere afrontar diversos retos, tales como la movilidad, e dinamismo y la heterogeneidad. Con el fin de dar cumplimiento a los requerimientos cada vez más exigentes de los usuarios, es necesario combinar varios servicios, lo cual mplica dotar a los ambientes ubicuos de mecanismos eficientes y eficaces de descubrimiento y composición. En el presente trabajo, se propone una plataforma de composicion de servicios para ambientes ubicuos, que considera la especificación del comportamiento de los servicios, el perfil contexto del usuario. De esta forma, es posible obtener un servicio compuesto, que satisfaga la demanda del usuario se ajuste a sus preferencias y al contexto en el cual se encuentra.

Palavras-chave: Ambientes ubicuos. Descubrimiento de servicios. Composición de servicios. Perfil de usuario. Contexto.

Abstract: Ubiquitous computing has established itself as the next generation of communications, offering to users the capabilities of the networks at any time and place, in order to support their daily tasks. However, the deployment of services for ubiquitous environments requires facing several challenges, such as mobility, dynamism and heterogeneity. To comply with the requirements of users, it is necessary to combine several services, which implies provide to ubiquitous environments of efich and discovery and composition. In this paper, we propose a service composition platform for ubiquitous environments, which considers the specification of the behavior of services, the userite profice that meets the demand of users and meets composite service that meets the demand of users and meets Keywords: Ubiquitous environments. Service discovery. Service composition. User profile. Context.
\end{abstract}

SUAREZ, Luis Javier: ROJAS, Luis Antonio; HERMIDA Victor Álberto; CAICEDO, Oscar Mauricio; CORRALES Juan Carlos. U-Bemp: Plataforma de Composicion de Servicios para Ambientes de Computacion Ubicua. Informática na Educação: teoria \& prática, Porto Alegre,
v. 14 , n. 1, p. 107-122, jan./jun. 2011 .

\author{
Luis Javier Suarez \\ Universidad del Cauca
}

Luis Antonio Rojas

Universidad del Cauca

Victor Alberto Hermida

Universidad del Cauca

\section{Oscar Mauricio Caicedo}

Universidad del Cauca

Juan Carlos Corrales

Universidad del Cauca

\section{Introducción}

$\mathrm{L}$

a computación ubicua puede ser descrita como dispositivos portables con facilidades embebidas de computación y comunicación, cuya meta es copar el ambiente con componentes electrónicos para ayudar de una manera natural a los usuarios durante sus tareas diarias (EL-SAYED; ABDUR-RAHMAN, 2006). Para lograr los objetivos de la computación ubicua, se requiere abordar retos significativos, principalmente relacionados con la heterogeneidad del ambiente, el dinamismo y la información que describe al usuario (perfil y contexto) (MOKHTAR, 2007), (SELLAMI et al., 2009). La computación ubicua debe soportar las tareas de los clientes por medio de la integración de las funcionalidades de los servicios de 
red, de tal forma, que éstos puedan ser invocados en cualquier momento, lugar y dispositivo de acceso (EL-SAYED; ABDURRAHMAN, 2006). Supongamos que un usuario necesita: i) un servicio de información turística que ofrezca información relacionada con los sitios de interés y restaurantes ubicados en la ciudad objeto de su visita. ii) Un reporte del clima para poder programar su recorrido en la ciudad. En este escenario, el turista realiza su petición por medio de una aplicación móvil y el ambiente de computación ubicua buscará los servicios de información sobre sitios turísticos, restaurantes y predicción del clima para poder crear un servicio compuesto que responda a sus exigencias.

Sin embargo, encontrar un servicio que cumpla exactamente con las exigencias de búsqueda del usuario se convierte en un caso excepcional, ya que la mayoría requieren de una adaptación de los servicios disponibles en la red o un proceso de composición de diversas capacidades para ejecutar tareas complejas (HERMIDA, 2009), (GRIGORI, 2010). Según la literatura, el mecanismo para combinar dos o más servicios disponibles y formar un nuevo servicio se denomina Composición de servicios. Esta composición puede ser estática o dinámica. Así, la primera es realizada durante la fase de despliegue, sin embargo, esta solución limita el posible uso de los recursos y no se adapta a las fluctuaciones propias de los ambientes ubicuos (ZHENGHUI et al., 2009). Mientras que la composición dinámica, busca combinar los servicios ubicuos disponibles en la red durante la fase de ejecución, brindando una mejor respuesta a los requisitos de heterogeneidad y dinamismo de los ambientes de computación ubicua (CORRALES, 2008),(BERARDI et al., 2005), (BROGI et al., 2008).

De este modo, en contextos con este tipo de características, es imprescindible contar con capacidades de descubrimiento y composición de servicios, que permitan explotar los recursos (aplicaciones, archivos, almacenamiento, hardware, etc.) presentes en la red. El descubrimiento y composición de servicios deben incorporar las características necesarias para ser tan dinámicos y autónomos como las condiciones de la red ubicua lo requieran.

En el presente trabajo se propone un mecanismo de descubrimiento y composición de servicios. La fase de descubrimiento se trata en extenso en (SUAREZ et al., 2011). Los algoritmos utilizados en dicha fase actúan sobre servicios descritos con BPEL (Business Process Execution Language) (TONY et al., 2003). Asimismo, las técnicas de descubrimiento consideradas, operan sobre información relacionada con el usuario, tal como su perfil y el contexto, con el objetivo de recuperar servicios que se ajusten a sus preferencias y puedan ser consumidos por el dispositivo móvil de acceso. El presente artículo, describe principalmente la etapa de composición de servicios, la cual se encarga de componer el proceso más similar al servicio requerido por el cliente, usando los servicios recuperados en la etapa de descubrimiento. Las técnicas utilizadas en esta fase, identifican de manera única las actividades involucradas en la composición. Posteriormente, analizan la conformidad de los servicios a componer, a través de la construcción de un grafo de dependencia de datos que permite establecer la compatibilidad de los servicios a partir de las interfaces. Luego, se obtiene la síntesis de composición, la cual esta soportada en el grafo de dependencia de datos, y el plan de la composición requerido.

En la siguiente sección, se presentan los trabajos relacionados. La sección 3, explica la arquitectura propuesta para la plataforma de descubrimiento y composición automática de servicios en ambientes de computación ubicua, denominada U-BeComp. En la sección 4 y 5 se describen las fases de descubrimiento y composición respectivamente. En la sección 6 se analizan los resultados obtenidos después de evaluar experimentalmente la plataforma. Por último, en la sección 7 se presentan las conclusiones y trabajos futuros.

\section{Trabajos Relacionados}

La composición de servicios se puede dividir en dos aspectos fundamentales, la síntesis y la orquestación (CORRALES, 
2008), (KÜSTER et al., 2005). La síntesis se refiere a cómo generar un plan para lograr el comportamiento deseado, a través de la combinación de múltiples servicios. La orquestación se encarga de la coordinación del flujo de control y datos entre varios componentes durante la ejecución del plan.

El presente artículo se enfoca exclusivamente en la síntesis autónoma de servicios, la orquestación de los servicios es un problema complementario que será abordado en trabajos futuros.

En (MOKHTAR, 2007) se propone una clasificación de soluciones para la composición de servicios basada en dos categorías: la composición basada en interfaces y la composición basada en conversaciones. La composición basada en interfaces es empleada cuando los servicios disponibles en la red y las tareas de usuario, son descritos como capacidades individuales sin una conversación asociada; en este modelo, los servicios son combinados basándose en la conformidad de sus signaturas (MOKHTAR, 2007). En esta categoría se pueden encontrar enfoques basados en búsquedas sobre grafos (ZHENGHUI et al., 2009), (HASHEMIAN; MAVADDAT, 2005) y soluciones soportadas en el encadenamiento de servicios (PONNEKANTI; FOX, 2002), (MARTÍNEZ; LESPÉRANCE, 2004), (MASUOKA et al., 2003), (RAMASAMY, 2006). En el grafo construido en (EL-SAYED; ABDUR-RAHMAN, 2006) los nodos representan los servicios disponibles y las aristas indican la correspondencia entre las salidas de un servicio y las entradas a otro. El problema de esta solución radica en su pobre escalabilidad cuando se incrementa el número de servicios contenidos en los repositorios, lo cual se ve explícito en su complejidad $O\left(n^{3}\right)$. El trabajo de (ZHENGHUI et al., 2009) presenta una aproximación para la agregación de servicios basada en grafos que permite la composición autónoma de servicios en ambientes ubicuos, construyendo un grafo de dos niveles, el primero representa las relaciones funcionales de los servicios y sus parámetros, mientras el segundo contiene las instancias de los servicios y los tipos de datos. La complejidad está determinada por
$O\left(m^{2}\right)$, donde $m$ representa una medida del tamaño de la ontología empleada para las comparaciones semánticas.

La composición de servicios basada en conversaciones, asume que los servicios poseen un comportamiento complejo, descrito a través de una conversación (CORRALES, 2008). La selección de conversaciones dirigida por objetivos, busca un comportamiento que responda a la tarea que el usuario requiere, en este caso no existe una integración con servicios que pertenezcan a otros procesos (BERNSTEIN; KLEIN, 2002). La integración de tareas dirigida por objetivos, selecciona un conjunto de conversaciones y las integra de tal forma que el servicio compuesto satisface la tarea de usuario, consumiendo las entradas proporcionadas y generando los efectos requeridos. En este enfoque, las tareas de usuario se expresan en términos de sus entradas y salidas, buscando una mayor facilidad en la formulación de los requisitos del usuario (BROGI et al., 2008), (SHIAA et al., 2008). La integración de conversaciones dirigida por conversaciones, captura el comportamiento en la tarea de usuario, y además, asume que tanto los servicios como las tareas de usuario son expresados por medio de una conversación (MOKHTAR, 2007), (BERARDI et al., 2005), (HASHEMIAN; MAVADDAT, 2005), los servicios compuestos se generan de acuerdo a la conversación definida en las tareas de usuario.

En (BERARDI et al., 2005) los autores presentan el framework Colombo, el cual aborda el problema de la composición automática de servicios web. Este trabajo incorpora la noción de "servicio meta", para denotar el comportamiento deseado para el servicio requerido. En (HASHEMIAN; MAVADDAT, 2005) los servicios y los requisitos de los usuarios son expresados como una terna ordenada compuesta por un conjunto de entradas, un conjunto de salidas y un conjunto de dependencias entre las entradas y las salidas. El conjunto de dependencias expresan el procesamiento que se desea para manejar los datos entregados por el usuario, este procesamiento puede ser realizado por uno o varios servicios atómicos. La composición 
es realizada por medio de la búsqueda en un grafo de dependencia. En (MOKHTAR, 2007) se presenta un mediador para la composición de servicios para ambientes ubicuos, en este trabajo las tareas de usuario son expresadas como una dupla conformada por un autómata que describe la conversación de la tarea y un conjunto de propiedades no funcionales que deben cumplir los servicios. La composición esta soportada en una representación de autómatas finitos y busca la integración de servicios, recuperados en una fase previa de emparejamiento semántico. La complejidad computacional de la solución está determinada por la función $O\left(m^{n b}\right)$, donde $n b$ es la cantidad de capacidades requeridas en la conversación y $\mathrm{m}$ es el número de capacidades contra el cual se compara la solicitud del usuario.

De las técnicas de composición expuestas, se tiene que el enfoque basado en conversaciones posee una mayor confiabilidad, al asegurar que intervienen los servicios requeridos por el usuario, dando así claridad sobre los datos que debe manipular cada uno de ellos, obteniendo un flujo de ejecución y de control similar al deseado. Por otro lado, la complejidad computacional de los anteriores trabajos no es la más adecuada en entornos tan dinámicos y heterogéneos como los ambientes de computación ubicua. De acuerdo a esto, el presente trabajo emplea una técnica de composición dirigida por conversaciones, que busca dar solución a la complejidad inherente a la composición de servicios. Así, para garantizar una mejor escalabilidad, las conversaciones solicitadas están compuestas por servicios atómicos, recuperados en la fase previa de descubrimiento, donde se encuentran los servicios más similares a los requeridos por el usuario.

\section{Arquitectura}

Esta sección presenta la arquitectura de la plataforma de descubrimiento y composición automática de servicios para

Figura 1 - Arquitectura de la plataforma U-BeComp

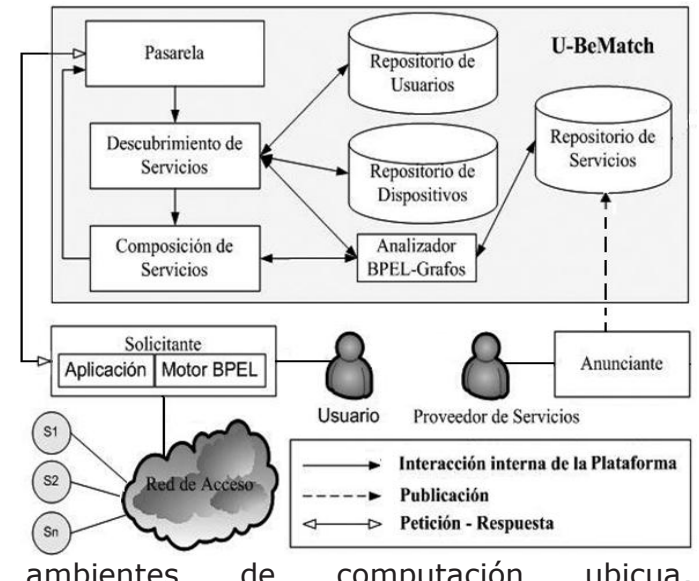

ambientes de computación ubicua, denominada U-BeComp. En el enfoque propuesto, tanto los servicios disponibles en la red ubicua, como las solicitudes del usuario son descritos mediante BPEL.

La Figura 1 describe brevemente U-BeComp y sus módulos. El usuario interactúa con la plataforma por medio de una aplicación, la cual soporta el envío de peticiones expresadas como modelos de comportamiento BPEL. El sistema recibe las solicitudes y las transforma a una representación formal de grafos, para posteriormente ejecutar el descubrimiento y la composición de servicios. El descubrimiento de servicios considera las preferencias de los usuarios móviles, las capacidades de los dispositivos de acceso y el contexto de entrega, con el objetivo de proveer flexibilidad para reconfigurar los servicios de acuerdo a los cambios del entorno. La composición está encargada de componer los servicios recuperados por el módulo de descubrimiento, con el fin de crear una conversación útil que ejecute las tareas de usuario establecidas en el requerimiento inicial.

Finalmente, la plataforma retorna al usuario el servicio compuesto, para que los servicios atómicos que lo conforman sean invocados por medio del motor BPEL. Por otro lado, los proveedores publican sus servicios como procesos BPEL, los cuales son almacenados en el Repositorio de Servicios y puestos a disposición para serconsumidos. Los módulos que componen a U-BeComp son detallados a continuación: 
Solicitante: módulo compuesto por una aplicación móvil y un motor BPEL. La aplicación ofrece la interfaz gráfica para la interacción con el usuario, además de proveer las capacidades de comunicación para el envío y recepción de los procesos BPEL. El motor BPEL es implementado por Sliver (HACKMANN et al., 2007), un mediador que soporta la ejecución de procesos BPEL en un amplio rango de dispositivos, desde computadores de escritorio hasta dispositivos móviles.

Pasarela: recibe las peticiones enviadas desde el Solicitante y transmite las repuestas generadas por los niveles superiores. Este componente brinda un acceso independiente de la red (Wifi, Bluetooth, etc.) y de los protocolos (HTTP, SMTP, SMS, etc.) a la plataforma.

Anunciante: ofrece las capacidades necesarias para que los proveedores publiquen sus servicios en el repositorio de la plataforma. Las descripciones de los servicios contienen atributos funcionales $y$ no-funcionales, tales como: entradas, salidas, precondiciones, restricciones, etc. Este módulo es implementado a través de una aplicación web.

Repositorios: almacenan información sobre la descripción de los servicios disponibles, las preferencias y el contexto del usuario. Estos repositorios son: i) Repositorio de Servicios: utiliza el repositorio de procesos de negocio presentado en (VANHATALO et al., 2006.), el cual soporta el almacenamiento y consulta de documentos BPEL ( $y$ otros documentos $\mathrm{XML}$ ). ii) Repositorio de Usuarios: almacena detalles relacionados con los usuarios, estos incluyen preferencias explicitas provistas por ellos (ej. Lenguaje nativo) o datos implícitos capturados dinámicamente tales como historial de servicios consumidos o patrones de uso y/o comportamiento. iii) Repositorio de dispositivos: almacena las características de los dispositivos tales como: capacidad de procesamiento, modalidades de presentación, interfaces de entrada, conectividad, etc. Esta información se recupera de los repositorios: UAProf (User Agent Profile) (W3C, 2001) y WURFL (Wireless Universal Resource) (PASSANI, 2007).

Analizador BPEL-Grafos: transforma las descripciones de comportamiento (BPEL) en su equivalente en grafos, implementando la estrategia presentada por (Mendling; Ziemann, 2005). El algoritmo emplea un proceso de transformación recursivo para cada tipo de actividad estructurada, tomando una aproximación de arriba-abajo (top-down). Las actividades básicas BPEL son transformadas en nodos y las secuencias son obtenidas conectando los nodos requeridos por medio de aristas. Las actividades estructuradas son representadas por medio de operadores lógicos XOR y AND.

Módulo de Descubrimiento de Servicios: recupera los servicios más apropiados para componer el modelo de comportamiento requerido por el usuario (SUAREZ et al., 2011).

Módulo de Composición de Servicios: encargado de componer el proceso más similar al servicio requerido por el cliente, usando los servicios recuperados en la etapa de descubrimiento. La composición se realiza teniendo en cuenta el flujo de control definido en la petición del usuario.

En las siguientes secciones, la fase de descubrimiento es descrita brevemente, mientras que el proceso de composición y las fases que lo conforman, como: la creación del grafo de dependencias y los procedimientos para realizar la síntesis de composición, son ampliamente detalladas.

\section{Fase de Descubrimiento}

En esta sección describiremos ligeramente el enfoque propuesto de la fase descubrimiento de servicios en ambientes de computación ubicua. El objetivo de esta etapa es proveer un mecanismo de recuperación de servicios, que tenga en cuenta tanto las preferencias del usuario, 


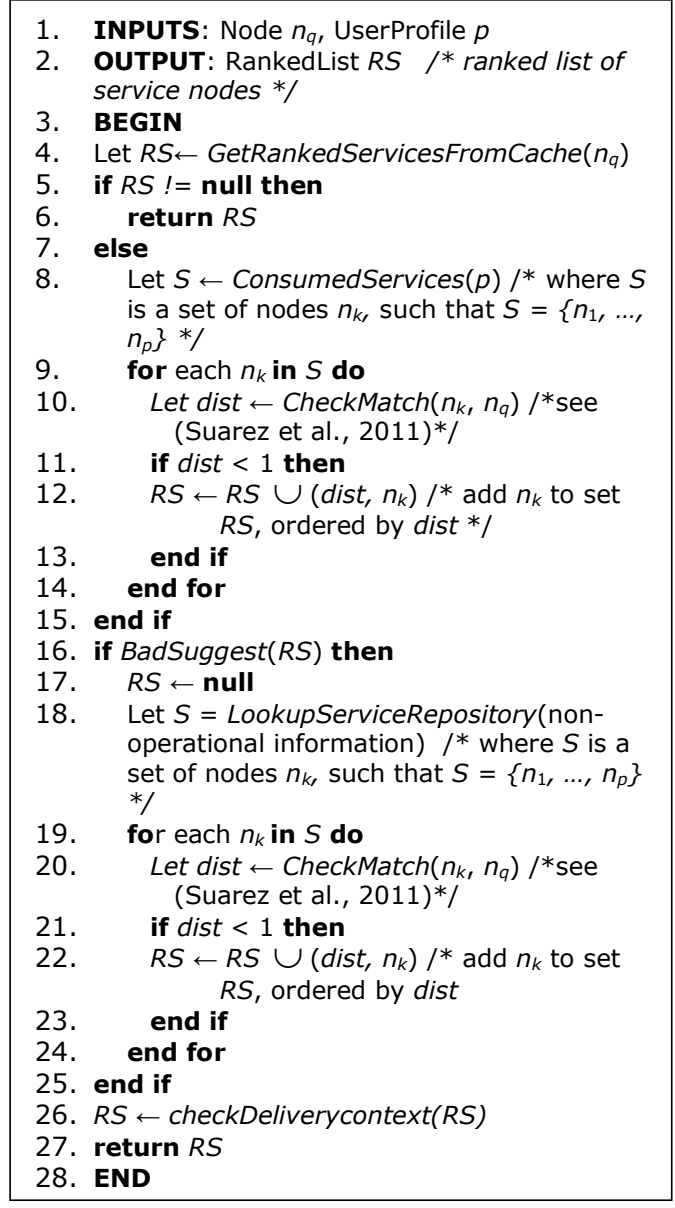

Algoritmo 1 - Función de Recuperación de Servicios

como las especificaciones de dispositivos y el contexto de entrega. El Algoritmo 1 define la función para obtener un conjunto ordenado de servicios, los cuales cumplen con la solicitud del usuario. Este algoritmo hace uso de dos funciones: La función GetRankedServicesFromCache $(n)$ provee una lista organizada de servicios desde la cache para cualquier solicitud $n$ del usuario (si dichos servicios existen). Si el usuario actual, u otros, no han enviado la solicitud $n$ previamente, el algoritmo recupera una lista de servicios, los cuales, el usuario actual u otros usuarios con perfiles similares, han invocado anteriormente. La función ConsumedServices $\left(p_{i}\right)$ retorna dichos servicios, donde pi es el perfil del usuario actual. El algoritmo obtendrá un servicio candidato, comparando el nodo de la solicitud del usuario ni contra cada servicio previamente invocado y lo adicionará a un lista organizada (ranking). CheckMatch $\left(n_{i,}\right.$ $n_{p}$ ) es una función que retorna un double, indicando la similitud semántica / distancia entre el nodo solicitado ni y el nodo candidato $\mathrm{np}$, para mayores detalles ver (SUAREZ et al., 2011).

La función BadSuggest(RS) retorna verdadero si un ranking dado de servicios RS, no contiene suficientes servicios que cumplan con un determinado umbral de similitud en contraste a la solicitud del usuario. En caso tal de no encontrar un servicio que cumpla satisfactoriamente con el requerimiento del usuario (es decir, BadSuggest retorna true), entonces todos los otros servicios registrados en el Repositorio de Servicios serán comparados con el servicio solicitado con el fin de producir el ranking deseado. LookupServiceRepository denota la función que permite recuperar los servicios desde el Repositorio de Servicios. Posteriormente, la función checkDeliverycontext(RS) verifica si los servicios recuperados pueden ser invocados en el contexto del usuario. Finalmente, se retorna un conjunto de los servicios recuperados, ordenado según el valor de similitud estimado respecto a los servicios de consulta.

\section{Fase de Composición}

El proceso de composición se inicia creando un grafo de dependencias, el cual permite establecer la compatibilidad entre las interfaces de los servicios que deben interactuar en la realización de la tarea de usuario. El grafo de dependencia es construido empleando el concepto de Hípergrafos (GALLO et al., 1993).

\subsection{Grafo de Dependencias}

El grafo de dependencia es construido empleando el concepto de Híper-grafos (GALLO et al., 1993). A continuación se definen formalmente los Híper-grafos.

Definición 1: Híper-grafo, es una dupla $\mathbf{H}=(\mathbf{V}, \mathbf{E})$, donde $\mathbf{V}=\mathbf{v}_{\mathbf{1}}, \mathbf{v}_{\mathbf{2}}, \mathbf{v}_{\mathbf{3}}, \ldots, \mathbf{v}_{\mathbf{n}}$ es el conjunto de vértices o nodos, y $\mathbf{E}=\mathbf{E}_{\mathbf{1}}, \mathbf{E}_{\mathbf{2}}$, $\mathbf{E}_{3}, \ldots, \mathbf{E m}$ con $\mathbf{E}_{\mathbf{i}} \subseteq \mathbf{V}$ para $\mathbf{i}=1,2,3, \ldots, \mathrm{m}$ es el conjunto de híper-aristas. Cuando $\left|\mathbf{E}_{\mathbf{i}}\right|=$ 
$2, \mathbf{i}=1,2, \ldots, \mathbf{m}$ el híper-grafo es una grafo convencional.

Definición 2: Híper-grafo dirigido, es un híper-grafo con híper-aristas dirigidas. Una híper-arista dirigida es una pareja ordenada $\mathbf{E}=(\mathbf{X}, \mathbf{Y})$, donde $\mathbf{X}$ es la cola de $\mathbf{E}$, denotada por $\mathbf{T}(\mathbf{E})$, mientras $\mathbf{Y}$ representa la cabeza $\mathbf{H}(\mathbf{E})$.

Definición 3: BF-Híper-grafo, un BFhíper-grafo, o simplemente $B F$-grafo, es un híper-grafo cuyas híper-aristas son del tipo B-arista o F-arista. Una B-arista (backward) es una híper-arista $\mathbf{E}=$ $((\mathbf{T}(\mathbf{E}), \mathbf{H}(\mathbf{E}))$ con $|\mathbf{H}(\mathbf{E})|=1)$. Una F-arista (forward) es una híper-arista $\mathbf{E}=$ $((\mathbf{T}(\mathbf{E}), \mathbf{H}(\mathbf{E}))$ con $|\mathbf{T}(\mathbf{E})|=1)$.

Para la construcción del grafo de dependencias, se emplean los nodos que representan las actividades recuperadas en la fase de descubrimiento y se crean nuevos nodos para representar los datos que conforman los mensajes de entrada y/o salidas de dichas actividades. Por tanto, las híper-aristas del grafo de dependencias poseen los siguientes tipos:

- $\quad \mathbf{E}_{\mathbf{p d}}=(\{\mathbf{p}\}, \mathbf{O})$. F-arista desde el nodo $p$ (tipo actividad) a un conjunto de nodos $O$ que contiene los datos de salida de $p$.

- $\mathbf{E}_{\mathbf{d p}}=(\mathbf{I},\{\mathbf{p}\})$. B-arista desde el conjunto de datos $I$ al nodo $p$ (tipo actividad), I representa el conjunto de datos de entrada de $p$.

- $\quad \mathbf{E}_{\mathbf{d d}}=(\{\mathbf{d}\}, \mathbf{D})$. F-arista desde el nodo $d$ (tipo dato) al conjunto de nodos $D$ (tipo datos), este tipo de híper-arista se utiliza para representar la asignación del valor de un dato a otro(s).

La construcción del Híper-grafo de dependencias es guiada por el grafo de consulta, una representación formal del documento BPEL requerido por el usuario. Este grafo está compuesto por una lista de nodos que representan las actividades básicas requeridas para ejecutar la conversación solicitada por el usuario; para su construcción se realiza un recorrido ordenado de los nodos de consulta y se van agregando los nodos que representan los datos de entrada y/o salida de los nodos de consulta, así como los nodos recuperados. A

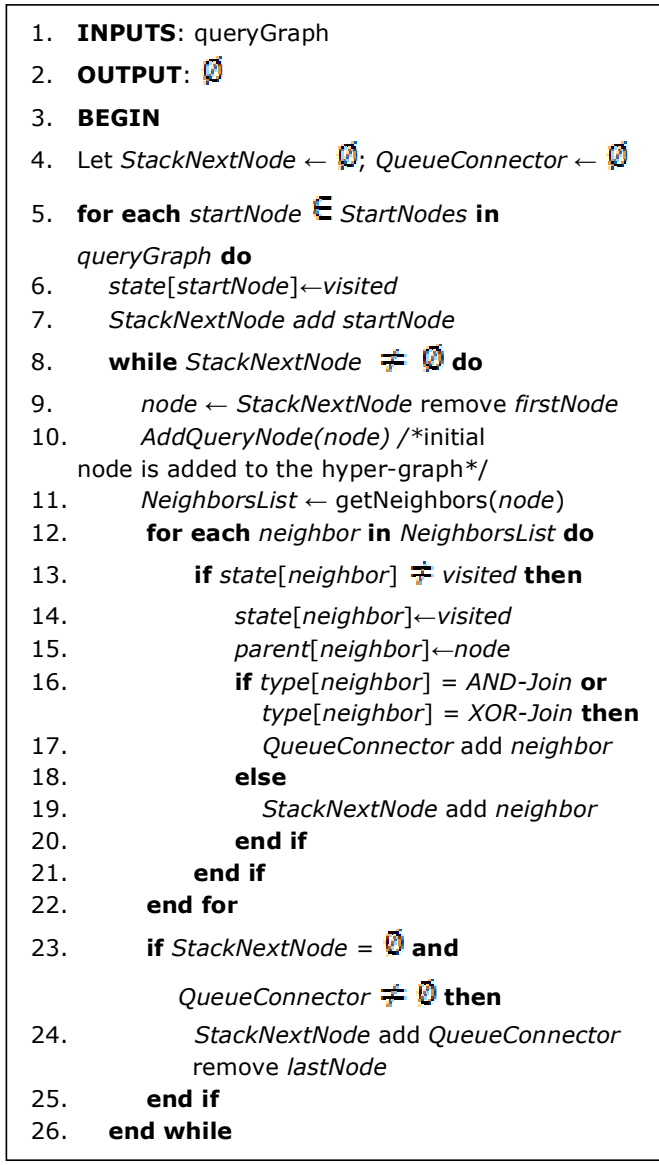

Algoritmo 2 - Función DFS Modificado

medida que se agregan los diferentes nodos se van creando las relaciones de dependencia a través de las híper-aristas que conectan los vértices. Los procedimientos para la construcción del Híper-grafo se describen a continuación.

\subsubsection{Construcción del Híper-grafo}

Este procedimiento describe el proceso de Recorrer el Grafo de Consulta. Así, la construcción del híper-grafo sigue el orden establecido en el grafo de consulta; se hace de esta forma para establecer las relaciones de dependencia de datos y ejecución entre las actividades básicas. El algoritmo de búsqueda en profundidad (DFS Depth First Search) es utilizado para recorrer el grafo de manera ordenada. El Algoritmo 2 describe la función DFS modificada para recorrer el grafo de consulta. El recorrido inicia por los nodos tipo Start contenidos en 


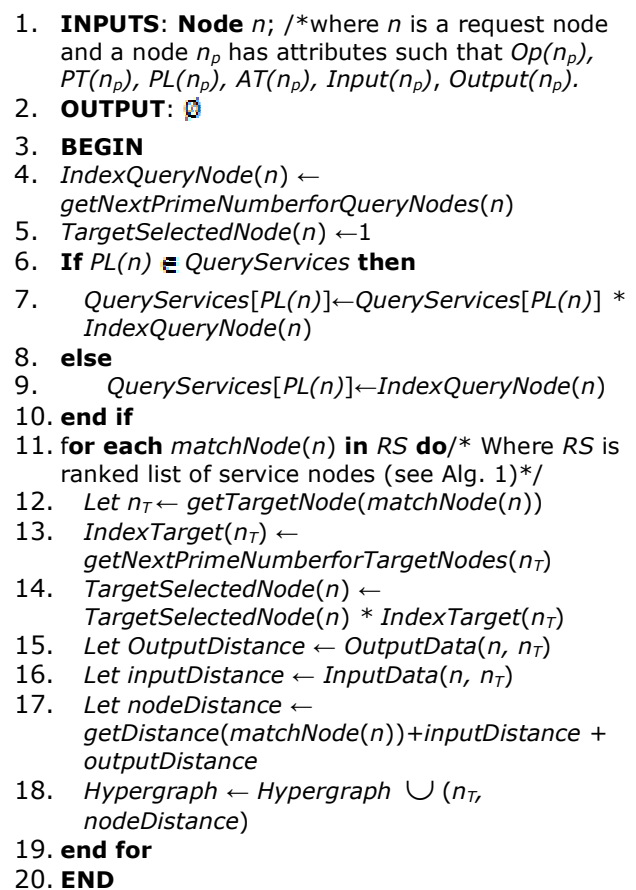

Algoritmo 3 - Función Adicionar Actividad de Consulta

el grafo de consulta (línea 6), agregando el nodo inicial al híper-grafo (línea 11), y colocando los nodos vecinos sujetos a procesamiento en una pila (línea 19). Cada nodo conserva el estado, esto quiere decir que a cada nodo procesado se le asigna el estado visitado (línea 15), con el fin de asegurar que el nodo sea procesado una sola vez. Este procedimiento es repetido por cada nodo que haya sido agregado a la pila (línea 8). Cada vez que se procesa un nodo se retira de la pila (línea 9). De manera alterna, una cola es construida (QueueConnector) para almacenar temporalmente los nodos tipo conector AND-Join y XOR-Join (línea 17), y así controlar la profundidad del recorrido. Encolar estos conectores en un arreglo diferente permite recorrer todas las ramas de una estructura compleja, antes de aumentar la profundidad más allá de los nodos donde convergen dichas ramas. Con esto se logra que al visitar un nodo determinado se hayan considerado todas aquellas actividades predecesoras que pertenecen a ramas concurrentes 0 excluyentes. El procedimiento para adicionar un nodo de consulta al híper-grafo de composición es detallado a continuación.

\subsubsection{Adicionar nodos de consulta al Híper-grafo}

Este procedimiento consiste en agregar los nodos tipo dato, que representan las entradas y/o salidas de la actividad básica de consulta, y sumar los nodos recuperados al híper-grafo creando las relaciones de dependencia entre los datos y las actividades predecesoras.

El Algoritmo 3, especifica cada uno de los pasos necesarios para adicionar un nodo de consulta al híper-grafo. Este algoritmo inicia asignando un código al nodo de consulta (línea 4), dicho código corresponde a un número primo único que identifica al nodo (el código es incrementado a medida que se agregan nuevos nodos). Posteriormente, las parejas entregadas por el módulo de descubrimiento (línea 11) son recorridas con el fin de agregar los nodos recuperados al híper-grafo. Cada nodo recuperado es identificado por un número primo único (línea 13) lo cual permite adicionarlo al híper-grafo (línea 18).

Mientras que los nodos recuperados son procesados, la comparación de las interfaces de entrada y salida de estos, respecto el nodo de consulta es llevada a cabo (línea 15 y 16). De esta manera son creadas las aristas de dependencias entre los datos y las actividades recuperadas. Si una actividad predecesora produce todos los datos que requiere una actividad subsecuente, se establece que son compatibles y que pueden participar en la composición.

\subsection{Síntesis de Composición}

La síntesis de composición selecciona el conjunto de servicios que serán empleados para realizar la tarea del usuario. Para ello, se debe seleccionar un conjunto de servicios que ofrezcan cada una de las operaciones requeridas en el proceso de negocio (tarea de usuario representada mediante un proceso descrito con BPEL) y cuyas interfaces sean compatibles. El Algoritmo 4, presenta el procedimiento para construir el árbol de servicios. Este algoritmo recibe 
1. INPUTS: ServicesMatches/*Let ServicesMatches $=\left\{s_{1}, \ldots, s_{n}\right\}$ where $s_{k}$ is a tuple of the form $S_{k}\left(n_{k}, N_{T}\right)$; such that $N_{T}=\left\{n_{T 1}, \ldots\right.$, $n_{T p}$ \}represents an ordered set

of services retrieved for the query node $n_{k}$.

2. OUTPUT: Tree

3. BEGIN

4. Let TreeDepth/*Number of Query Services*/

5. Let Depth $\leftarrow 0$

6. Let Tree $\leftarrow \Phi / *$ Composition Tree*/

7. Let TreeIndexXOR $\leftarrow 1 /{ }^{*}$ this index is the product of the indexes of those services that are not compatible with the services added in the composition. */

8. Let TreeIndexAND $\leftarrow 1{ }^{*}$ this index is the product of the indexes assigned to the services used in the composition */.

9. While Depth <TreeDepth do

10. Let serviceRetrieved $\leftarrow$ getServiceMatchForQueryService(Depth)

11. If serviceRetrieved $\neq 0$ then

TreeaddMatchServiceIn (Position $\leftarrow$ Depth) $/ *_{\text {if }}$ this function finds a service that is compatible it is added to the tree, including its composition indices $X O R$ and AND in order to establish what services can be added later*/

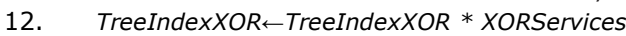
[serviceRetrieved]

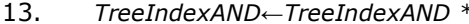
TargetService[serviceRetrieved]

14. Depth++

15. else

16. Depth $\leftarrow$ Depth $-1 /^{*}$ this is when a retrieved service is not found, which is compatible with previously selected., in this case it returns a level in the tree to select a new service*/

17. If Depth $>=0$ then /*If the depth of the tree is greater than zero, the node is removed and removed its composite index $X O R$ and $A N D *$ /

18. RemovedService $\leftarrow$ Tree get node at Depth

19. TreeIndexXOR TreeIndexXOR / XORServices [RemovedService]; TreeIndexAND TreeIndexAND / TargetService[RemovedService]

20. else

21. return $\delta / *$ When the depth is less than zero is not possible to perform the services composition */

22. end if

23. end while

24. return Tree $/ *$ this is the successful case, the tree represents a composition*/

25. END

Algoritmo 4 - Función Construir Árbol de Servicios

como entrada los servicios de consulta, junto con el conjunto de servicios recuperados para cada uno de ellos (ServicesMatches). La construcción del árbol tiene como objetivo seleccionar un servicio recuperado $n_{T k}$ que pertenece a $N_{T}=\left\{n_{T 1}, \ldots\right.$, $\left.n_{T p}\right\}$ por cada servicio de consulta $n_{k}$ (ver Algoritmo 4, línea 1), para esto se van adicionando los servicios recuperados de una manera ordenada de acuerdo a su similitud con el servicio de consulta. La función getServiceMatchForQueryService() (línea 10) escoge de manera ordenada el servicio recuperado que debe ser adicionado al árbol. Esta selección toma en cuenta la compatibilidad del servicio recuperado con los servicios adicionados previamente. El algoritmo es ejecutado hasta que un servicio recuperado es agregado por cada servicio de consulta, esta condición asegura que todos los elementos para componer el proceso de consulta son considerados. Una vez terminada la selección de servicios, se procede a generar un proceso de negocio abstracto a partir de ellos. Este proceso es realizado siguiendo el comportamiento especificado en la tarea requerida por el usuario.

\section{Ejemplo de Composición}

Considere un escenario de flujo de datos como el que se plantea en la Figura 2, en el grafo de consulta se define un intercambio de datos (departure y return) entre dos actividades ClientRequest y AirlineReservation.

En el lado derecho de la gráfica se representa la conformación del híper-grafo con los nodos recuperados y los nodos tipo dato. En primera instancia se puede observar que a cada nodo se le ha agregado un atributo índice, para los nodos de consulta se utiliza una serie de números primos y para los nodos recuperados otra.

La Tabla 1 contiene los valores de índices para los nodos tipo actividad básica contemplados en el ejemplo. El valor obtenido para el índice TargetSelected se asocia directamente con el nodo de consulta correspondiente, y es equivalente al producto de los índices asignados a los nodos recuperados para dicho nodo de consulta. Cuando se agregan los nodos recuperados para la actividad de consulta ClientRequest se realiza la comparación de las interfaces de entrada. Se contrastan las salidas de la actividad ClientRequest con las salidas de ClientRequestA, ClientRequestB y ClientRequestC. 


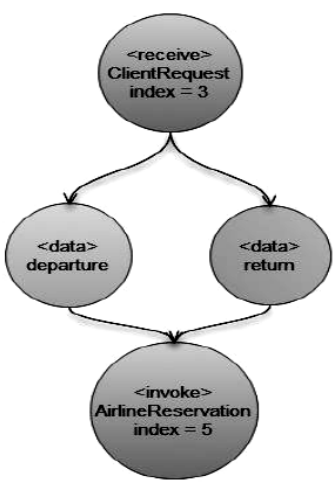

(a)

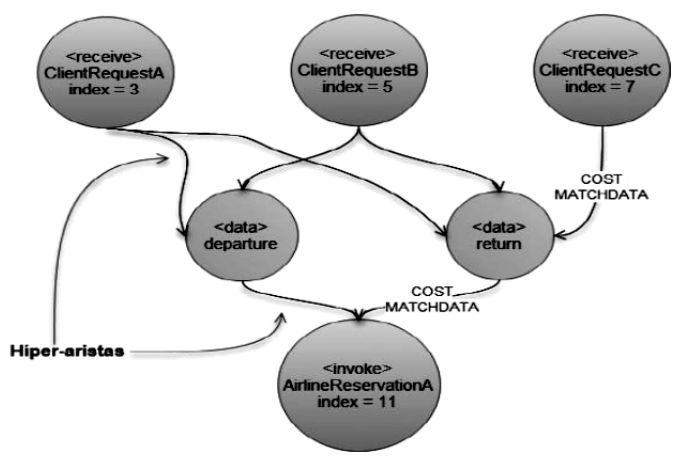

(b)

Figura 2 - Ejemplo Flujo de Datos: (a) Grafo de Consulta. (b) Híper-Grafo de Dependencias

Tabla 1 - Valores de Índices para las Actividades Básicas

\begin{tabular}{|l|l|l|}
\hline $\begin{array}{c}\text { Índice Nodos } \\
\text { de Consulta }\end{array}$ & $\begin{array}{c}\text { Índice Nodos } \\
\text { Recuperados }\end{array}$ & $\begin{array}{c}\text { Índice } \\
\text { TargetSelected }\end{array}$ \\
\hline ClientRequest $=3$ & $\begin{array}{l}\text { ClientRequestA }=3 \\
\text { ClientRequest } B=5 \\
\text { ClientRequestC }=7\end{array}$ & $\begin{array}{l}\text { ClientRequestA } * \\
\text { ClientRequest } B \\
\text { ClientRequestC } \\
=3 * 5 * 7=105\end{array}$ \\
\hline $\begin{array}{l}\text { AirlineReservatio } \\
n=5\end{array}$ & $\begin{array}{l}\text { AirlineReservationA } \\
=11\end{array}$ & \\
\hline
\end{tabular}

Cuando se encuentra una salida equivalente en las actividades recuperadas se agrega una híper-arista entre la actividad híper-una híper-arista entre la actividad recuperada y el dato de consulta. Los índices contenidos en la Tabla 2, son valores asignados a los datos de salida de la actividad ClientRequest al momento de agregarlos al híper-grafo.

Este proceso inicia realizando el emparejamiento de los datos de entrada de los dos nodos (de consulta y recuperado) obteniendo la medida de su similitud. Terminado el emparejamiento se verifica si todas las entradas del nodo recuperado tienen un par correspondiente en los datos del nodo de consulta, si esta condición no se cumple, se entiende que al menos uno de los datos necesarios para la invocación del nodo recuperado no estará presente en el proceso compuesto, por lo que esta operación no podrá ser ejecutada y se le asignará una distancia equivalente a $\infty$, indicando que este nodo debe ser ignorado al momento de realizar la composición de servicios. Si todas las entradas del nodo recuperado fueron emparejadas, se recorren las parejas encontradas para crear la relación entre el nodo recuperado y los datos de entrada del nodo de consulta. Si se encuentra una pareja de datos con una distancia de similitud igual a 1 se concluye que la actividad recuperada no tiene como entrada uno de los datos requeridos en la operación de consulta, bajo esta condición se declara al nodo como no ejecutable bajo las restricciones expresadas en el proceso de negocio y se lo descarta para una posible composición. Si se cumplen todas las condiciones de compatibilidad entre los datos de los dos nodos comparados se crea una híper-arista (ver Figura 2) para representar la relación entre los datos de entrada y el nodo recuperado, además, se le asigna un peso a la híper-arista equivalente a la similitud estimada por la función de emparejamiento de mensajes y un atributo denominado MatchData que indica con que dato del nodo recuperado fue emparejado el dato de consulta.

El proceso descrito crea un conjunto de dependencias denominado DataFlow, en el cual se define una memoria con el fin de identificar qué datos de entrada deben ser producidos por una misma actividad predecesora. Esto es importante para conocer qué actividades agregadas previamente generan datos compatibles con las entradas requeridas por el nodo recuperado que se está procesando, permitiendo deducir cuáles pueden ser combinadas con dicho nodo y cuáles no.

Para esto, se inicia averiguando el índice del nodo de consulta predecesor (QNP) que genera el dato de entrada (queryTail), este índice se establece cuando se agregan los datos de salida del nodo de consulta, luego se verifica si ese nodo predecesor ya fue 
incluido en el flujo de datos, si es así, quiere decir que existen otros datos de entrada que son generados por ese nodo QNP. Ahora se debe identificar cuáles nodos recuperados para el nodo QNP producen todos los datos requeridos como entrada, esto se obtiene calculando el máximo común divisor (MCD) de los índices (TargetDataTail) asignados a los nodos tipo dato. El presente ejemplo aclara el funcionamiento de lógica descrita.

Tabla 2 - Valores de Índices para las Actividades Básicas

\begin{tabular}{|l|c|l|}
\hline Nodo Dato & QueryDataTail & \multicolumn{1}{|c|}{ TargetDataTail } \\
\hline departure & 3 (ClientRequest) & $\begin{array}{l}\text { ClientRequestA } * \\
\text { ClientRequestB }=3 * 5 \\
=15\end{array}$ \\
\hline return & 3 (ClientRequest) & \\
\hline
\end{tabular}

Para los datos se tiene que el índice QueryDataTail es igual a 3, correspondiente al índice asignado a la actividad de consulta (QNP) que los genera en el proceso de consulta. El índice TargetDataTail para el dato departure es 15, producto de la multiplicación de los índices de las actividades recuperadas que generan dicho dato (ClientRequestA y ClientRequestB), mientras para el dato return el TargetDataTail es 35 (ClientRequestB * ClientRequestC).

Cuando se agrega la segunda actividad del ejemplo (AirlineReservation) se utilizan los índices generados previamente al agregar las actividades recuperadas y el emparejamiento de datos. Como primera medida se adicionan los nodos recuperados para la actividad de consulta AirlineReservation y se establece la similitud con los datos de entrada establecidos en el flujo de consulta, para este caso, constituye la relación de los datos departure y return con la actividad AirlineReservationA. Una vez creadas las híper-aristas entre los datos $y$ el nodo recuperado, ver Figura 2, se procede a determinar la compatibilidad de la actividad AirlineReservationA con las actividades que generan sus datos de entrada, en este caso ClientRequestA, ClientRequestB y ClientRequestC.

En primer lugar se identifican qué datos proceden de una misma actividad predecesora, esto se hace comparando los índices QueryDataTail asignados a los datos. En el ejemplo propuesto los datos departure y return poseen el mismo valor de QueryDataTail, lo cual indica que proceden del nodo de consulta ClientRequest. Para efectos de la composición de servicios, se debe averiguar cuáles de las actividades recuperadas producen estos dos datos, esto se realiza obteniendo el MCD de los índices TargetDataTail asignados a los datos cuando se relacionaron como salidas de los nodos recuperados en el híper-grafo. Para el ejemplo se tendría lo siguiente:

- TargetDataTail[departure] $=15$

- TargetDataTail[return]=105

- $\operatorname{MCD}($ TargetDataTail[departure], TargetDataTail[return] $)=15$

EI MCD calculado representa el producto de los índices de los nodos recuperados que generan las entradas requeridas por el nodo que se está procesando. Para este caso, se tiene que las actividades ClientRequestA y ClientRequest $B$ producen las dos entradas que requiere la actividad AirlineReservation, por tal motivo son compatibles a nivel de sus interfaces. Por el contrario, la actividad ClientRequestC es descartada ya que no genera uno de los datos requeridos para invocar la actividad procesada. Con estos datos se puede calcular matemáticamente qué actividades predecesoras no son compatibles a nivel de interfaces, para lo cual se debe obtener el conjunto de actividades recuperadas predecesoras (ClientRequestA, ClientRequestB y ClientRequestC), consultando el índice TargetSelected de la actividad QNP. Seguido, se procede a dividir el TargetSelected con el MCD calculado para los índices TargetDataTail de los datos, el resultado de esta división representa los índices de los nodos que no son compatibles a nivel de datos con el nodo que se está evaluando. De acuerdo a esto se tiene:

- TargetSelected[ClientRequest] $=105$

- MCD(TargetDataTail[departure], TargetDataTail[return]) $=15$

- TargetSelected[ClientRequest] $/ \mathrm{MCD}=$ $105 / 15=7$

En el ejemplo se tiene que la actividad recuperada con índice 7 es incompatible con la operación AirlineReservation, tal como se 
había resaltado anteriormente. Estos índices de incompatibilidad son almacenados en un registro con el fin de determinar posteriormente, durante la síntesis, qué servicios pueden ser combinados y cuáles no.

En resumen, el procedimiento para agregar una actividad básica crea una estructura en el híper-grafo, en la cual se relacionan las actividades recuperadas con los datos especificados en el proceso de consulta. Esto determina en primera instancia qué actividades recuperadas producen o consumen los datos requeridos, y cuáles actividades se pueden combinar de acuerdo a sus interfaces para llevar a cabo la composición de servicios.

\section{Experimentación}

Esta sección presenta el estudio experimental de la plataforma propuesta para la composición de servicios en ambientes de computación ubicua. El algoritmo de composición se implementó en lenguaje Java y los experimentos fueron realizados en un computador con procesador Pentium 4 2,30GHz, 1.000 MB de RAM bajo el S.O. Linux, Ubuntu.

Para evaluar los algoritmos definidos y determinar la eficiencia de los mismos, se caracterizaron los tiempos de respuesta según el estudio realizado en (Joines et al., 2002), donde: $r$ es el tiempo de respuesta en segundos; de tal forma que $r$ es clasificado como: Optimo cuando $r \leq 0.1$, Bueno cuando $0.1 \leq r \leq 1$; Aceptable cuando $1 \leq r \leq 10$; y Deficiente cuando $r \geq$ 10. La evaluación de rendimiento fue realizada sobre los principales módulos que implementan las funcionalidades de la fase de composición, tales como: la conformidad de los servicios y la síntesis de la composición. De esta manera, se definieron dos casos de prueba que determinan el tiempo que tarda el módulo de composición en entregar un resultado ante una tarea de consulta, cuando: el número de servicios de consulta varía, manteniéndose constante el número servicios recuperados, y cuando se varia el número de servicios recuperados y la cantidad de servicios que conforman el proceso de consulta se mantiene constante.
En este orden de ideas, se conformó un banco de pruebas (repositorio de procesos de negocio (VANHATALO et al., 2006.)) compuesto por documentos BPEL, distribuidos en cinco dominios relacionados con procesos de información turística: CarRental, Guesthouse, Holidays, Hotel y Travel. Estos procesos BPEL suman un total de 148 actividades básicas, las cuales representan los servicios presentes en la red ubicua y pueden ser consideradas como operaciones publicadas/disponibles para ser empleadas en las fases de descubrimiento y composición. Por tanto, para el primer caso de evaluación, se contó con 18 archivos BPEL de consulta, en los cuales se incrementó gradualmente el número de actividades básicas contenidas en dichos procesos BPEL. Este incremento significó un aumento en el número de datos que se emplearon, tal como se aprecia en la Tabla 1 , la cual expone el cálculo del número total de nodos procesados por el algoritmo de composición, al momento de crear el hípergrafo de acuerdo a los parámetros de entrada de los documentos BPEL de consulta. Este número total corresponde a la suma de la cantidad de actividades básicas y el número de datos que se declaran en el documento BPEL.

Tabla 3 - Conformación de los Documentos BPEL de Consulta

\begin{tabular}{|c|c|c|c|}
\hline BPEL & $\begin{array}{c}\text { Actividades } \\
\text { Básicas }\end{array}$ & Datos & $\begin{array}{c}\text { Total } \\
\text { Nodos }\end{array}$ \\
\hline 1 & 3 & 8 & $\mathbf{1 1}$ \\
\hline 2 & 4 & 10 & $\mathbf{1 4}$ \\
\hline 3 & 5 & 14 & $\mathbf{1 9}$ \\
\hline 4 & 6 & 30 & $\mathbf{3 6}$ \\
\hline 5 & 7 & 27 & $\mathbf{3 4}$ \\
\hline 6 & 8 & 36 & $\mathbf{4 4}$ \\
\hline 7 & 9 & 36 & $\mathbf{4 5}$ \\
\hline 8 & 10 & 20 & $\mathbf{3 0}$ \\
\hline 9 & 11 & 44 & $\mathbf{5 5}$ \\
\hline 10 & 12 & 47 & $\mathbf{5 9}$ \\
\hline 11 & 13 & 50 & $\mathbf{6 3}$ \\
\hline 12 & 14 & 30 & $\mathbf{4 4}$ \\
\hline 13 & 15 & 34 & $\mathbf{4 9}$ \\
\hline 14 & 16 & 53 & $\mathbf{6 9}$ \\
\hline 15 & 17 & 56 & $\mathbf{7 3}$ \\
\hline 16 & 18 & 56 & $\mathbf{7 4}$ \\
\hline 17 & 19 & 59 & $\mathbf{7 8}$ \\
\hline 18 & 20 & 62 & $\mathbf{8 2}$ \\
\hline & & & \\
\hline
\end{tabular}

Para el primer caso de prueba, se ingresaron los documentos de consulta a la aplicación de descubrimiento y composición, y se midió el tiempo que tarda en realizar las tareas de composición. Así, por cada 


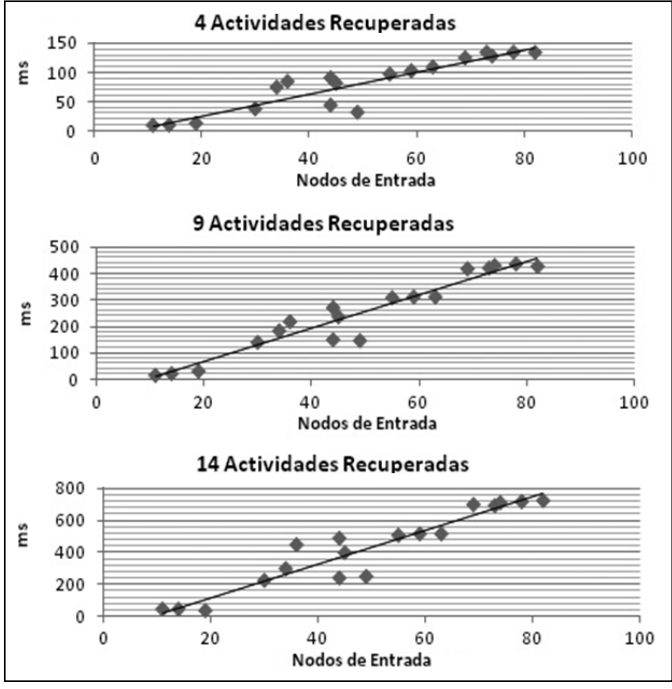

Figura 3. Gráficas de Rendimiento del Algoritmo de Composición en Función de los Nodos de Entrada

documento de consulta se tomaron 5 muestras de tiempos con el fin de establecer la media y obtener una aproximación para el tiempo de ejecución. Dichas pruebas fueron realizadas en diferentes escenarios, de acuerdo al número de actividades que se deseaban recuperar del repositorio de procesos, variando el número $K$ de actividades recuperadas.

En la Figura 3 se muestran las gráficas de comportamiento obtenidas de la aplicación de composición para tres casos, cuando se recuperan 4, 9 y 14 actividades del repositorio de procesos. Las gráficas presentadas en la Figura 3, evidencian que el tiempo de respuesta de los algoritmos de composición varía linealmente a medida que se incrementan la cantidad de actividades básicas y los datos que pertenecen al proceso de consulta. Para dichos escenarios de evaluación (4 a 14 actividades recuperadas), la relación del tiempo de respuesta contra los nodos de entrada se puede generalizar como una recta: $\boldsymbol{t}=\boldsymbol{m} * \boldsymbol{q}$ $+\boldsymbol{C}$; donde $\boldsymbol{t}$ es el tiempo, $\boldsymbol{m}$ la pendiente de la recta, $\boldsymbol{q}$ los nodos de consulta y $\boldsymbol{C}$ una constante. La pendiente $(m)$ de la recta varía de acuerdo a la cantidad de actividades recuperadas, entre mayor sea el número de actividades recuperadas, mayor es la pendiente.

Según la caracterización realizada en (JOINES et al., 2002), se determinó los siguientes tipos de comportamiento: Óptimo (tiempo de respuesta menor o igual a $0,1 s$.$) - cuando el número de nodos de$ consulta es inferior a 20. Esto se mantiene para todos los escenarios donde se varió el número de actividades recuperadas. Bueno (tiempo de respuesta entre $0,1 \mathrm{~s}$. y $1 \mathrm{~s}$.) - en esta categoría se encuentra el comportamiento del algoritmo para documentos de consulta que posean una cantidad de nodos de entrada (actividades básicas y datos) entre 20 y 82 . Así, los escenarios de evaluación, con distinto número de actividades recuperadas, alcanzaron tiempos de respuesta inferiores a 1 segundo en la ejecución de los algoritmos de composición.

Para el segundo caso de estudio, se determina el comportamiento de los algoritmos de composición, variando el número de actividades básicas que conforman el documento BPEL de consulta. Estas pruebas de rendimiento fueron realizadas con 18 archivos (ver Tabla 3 ).

Lo anterior, permite evaluar los tiempos de respuesta de los algoritmos a medida que se aumenta el número de servicios recuperados en un rango de 3 a 14 actividades, manteniendo constante las actividades y datos que los componen. Así, para cada incremento en el número de servicios recuperados, se toma varias muestras de tiempo, las cuales son promediadas para obtener un tiempo estimado de ejecución.

Lo anterior es expuesto en la Figura 4, donde se muestran las gráficas obtenidas del comportamiento de la aplicación de composición para tres casos, procesos BPEL de consulta de 11, 59 y 82 nodos de entrada (entre actividades básicas y datos). Por tanto, las gráficas presentadas en la Figura 3, exponen el tiempo de respuesta de los algoritmos de composición, el cual varía linealmente a medida que se incrementan la cantidad de actividades básicas recuperadas. Para los escenarios evaluados (11 a 82 nodos de entrada), la relación del tiempo de respuesta contra las actividades recuperadas se puede generalizar igualmente como una recta: $\boldsymbol{t}=\boldsymbol{p} * \boldsymbol{k}+\boldsymbol{C}$; donde $\boldsymbol{t}$ representa el tiempo de respuesta, $\boldsymbol{p}$ la pendiente de la recta, $\boldsymbol{k}$ los nodos 
recuperados y $\boldsymbol{C}$ una constante. De esta forma, la pendiente $(p)$ de la recta varía linealmente de acuerdo a la cantidad de actividades y datos de entrada.
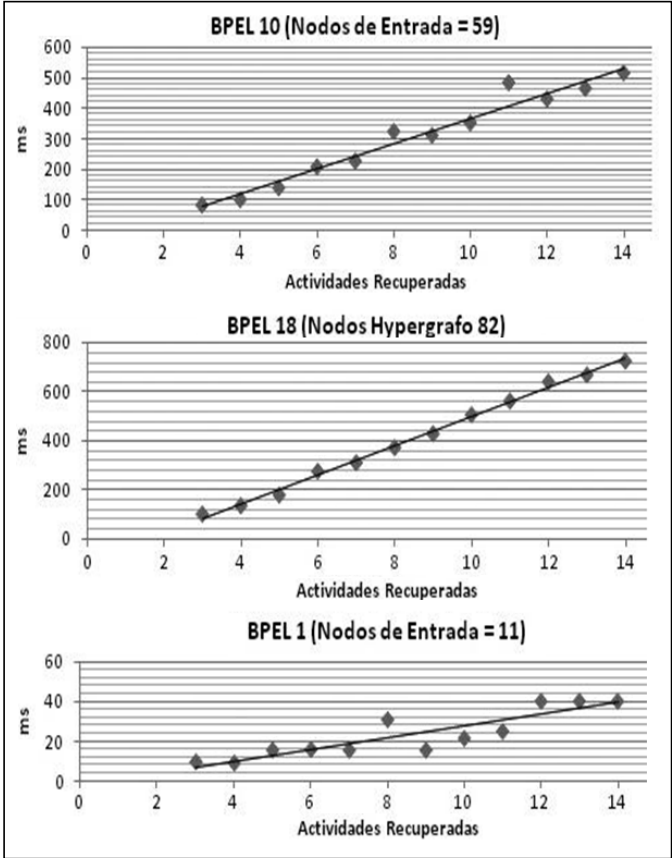

Figura 4. Gráficas de Rendimiento del Algoritmo de Composición en Función de las Actividades Recuperadas

\section{Conclusiones}

El enfoque presentado usa técnicas de emparejamiento y composición que trabajan sobre modelos de comportamiento. Se realiza una evaluación de la distancia semántica entre las actividades requeridas por el usuario y aquellas contenidas en el repositorio de la plataforma, soportando la entrega de aproximaciones cuando no se obtiene una coincidencia exacta de los parámetros de búsqueda. El problema del emparejamiento de actividades y composición de servicios es abordado por medio de una representación formal de grafos, que permite extraer las actividades básicas requeridas y obtener un formalismo para el proceso de composición de las actividades recuperadas.
Considerando el comportamiento de los algoritmos de composición frente a variaciones en la cantidad de actividades de consulta y el número de actividades recuperadas que entrega el módulo de descubrimiento, se puede concluir que la complejidad de la solución de composición está dada por: $t=q^{*} k$; donde $q$ es el número de actividades y datos de consulta, y $k$ el número de actividades recuperadas. Así, la complejidad computacional de los módulos de descubrimiento y composición está determinada por el producto del número de actividades de consulta y la cantidad de actividades recuperadas, expresado como $O(n)$, comportamiento esperado, y adecuado para entornos de computación ubicua, ya que los algoritmos realizan comparaciones uno a uno de las actividades que pertenecen a los dos conjuntos. El comportamiento lineal de la presente solución evidencia una diferencia significativa con respecto a los trabajos citados en la sección 2 (MOKHTAR, 2007), (ZHENGHUI et al., 2009), (BERARDI et al., 2005), (HASHEMIAN; MAVADDAT, 2005), los cuales poseen una complejidad exponencial. El número de comparaciones es reducido específicamente en la fase de descubrimiento, por medio del filtrado de candidatos, basado en el tipo de actividad, dominio de los procesos de negocio y el contexto de entrega del usuario, y por la reducción del espacio de búsqueda, gracias a los perfiles de usuario. Como trabajo futuro se plantea abordar la fase de orquestación de la composición, la cual permitirá realizar un control dinámico de las síntesis de composición generadas durante la fase de ejecución. Con esto es posible obtener capacidades de reconfiguración de los planes de composición sobre la marcha, una vez iniciada la ejecución de las tareas de usuario.

\section{Referencias}

BERARDI, D.;D. CALVANESE;G. D. GIACOMO;R. HULL;M. MECELLA. Automatic Composition of Web Services in Colombo. Thirteenth Italian Symposium on Advanced Database Systems, SEBD. BrixenBressanone, Italy, 2005. 
BERNSTEIN, A.;M. KLEIN. Towards high-precision service retrieval. ISWC, LNCS. Sardinia. Italia. 2342: 84-101, 2002.

BROGI, A.;S. CORFINI;R. POPESCU. Semantics-based composition-oriented discovery of Web services. ACM Transactions on Internet Technology (TOIT). 8, 2008.

CORRALES, J. C. Behavioral matchmaking for service retrieval. 2008, tesis presentada a la University of Versailles Saint-Quentin-en-Yvelines para optar al grado de Doctor of Philosophy in Sciences, Versailles, France.

DANIELA GRIGORI, J. C. C., MOKRANE BOUZEGHOUB, AHMED GATER. Ranking BPEL Processes for Service Discovery. IEEE Transactions on Services Computing, 2010.

EL-SAYED;ABDUR-RAHMAN. Semantic-based context-aware service discovery in pervasive-computing environments. Proceedings of 1st IEEE Workshop on Service Integration in Pervasive Environments. Lyon, France, 2006.

GALLO, G.;G. LONGO;S. NGUYEN;S. PALLOTTINO. Directed hypergraphs and applications. Discr. Appl. Math. 2: 177-201, 1993

HACKMANN, G.;C. GILL;G. C. ROMAN. Extending BPEL for interoperable pervasive computing. ICPS. . I. C. S. Press. Istanbul: 204-213, 2007

HASHEMIAN, S. V.;F. MAVADDAT. A Graph-Based Approach to Web Services Composition. The 2005 Symposium on Applications and the Internet (SAINT'05), 2005.

HERMIDA, V., O. CAICEDO, J.C. CORRALES, D. GRIGORI, AND M. BOUZEGHOUB. Service Composition Platform for Ubiquitous Environments Based on Service and Context Matchmaking, Bucaramanga, Colombia, Cuarto Congreso Colombiano de Computación 4CCC, Bucaramanga, 2009.

JOINES, S.;R. WILLENBORG;K. HYGH. Performance Analysis for Java Websites, Addison-Wesley, ISBN-13: 978-0201844542, 2002.

KÜSTER, U.;M. STERN;B. KÖNIG-RIES. A classification of issures and approaches in service composition. In Proceedings of the First International Workshop on Engineering Service Compositions (WESC05). Amsterdam, Netherlands, 2005.

MARTÍNEZ, E.;Y. LESPÉRANCE. Web service composition as a planning task: Experiments using knowledgebased planning. the 14th Int. Conf. on Automated Planning and Scheduling (ICAPS 2004). Whistler, BC, Canada, 2004.

MASUOKA, R.;B. PARSIA;Y. LABROU. Task computing the semantic web meets pervasive computing. 2nd International Semantic Web Conference (ISWC2003), 2003.

MENDLING, J.;J. ZIEMANN. Transformation of bpel processes to epcs. Proc. of the 4th GI Workshop on Event-Driven Process Chains (EPK2005). Hamburg. Germany. 167: 41-53, 2005.

MOKHTAR, S. B. Semantic Middleware for Service-Oriented Pervasive Computing. 2007, These(DOCTEUR) Devant L'universit' e de Paris 6, Paris, Francia

PASSANI, L. (2007). 7 de octubre de 2010. "Wurlf." from http://wurfl.sourceforge.net/.

PONNEKANTI, S. R.;A. FOX. SWORD: A developer toolkit for web service composition. The 11th Int. WWW Conf. (WWW2002). Honolulu, HI, USA 2002.

RAMASAMY, V. Syntactical and semantical web services discovery and composition. The 3rd IEEE International Conference on Enterprise Computing, E-Commerce, and E-Services (CEC/EEE'06), 2006.

SELLAMI, M.;S. TATA;B. DEFUDE. Service Discovery in Ubiquitous Environments: Approaches and Requirement for Context-Awareness. In Advances in Semantics for Web services Workshop, Milan, Italy, BPM Workshops, 2009.

SHIAA, M. M.;J. O. FLADMARK;B. THIELL. An Incremental Graph-based Approach to Automatic Service Composition. IEEE International Conference on Services Computing. Washington, DC, USA 2008.

SUAREZ, L. J.;L. A. ROJAS;]. C. CORRALES;L. A. STELLER. Service Discovery in Ubiquitous Computing Environments The Sixth International Conference on Internet and Web Applications and Services (ICIW 2011) St. Maarten, The Netherlands Antilles, 2011. 
TONY, A., et al. "Business Process Execution Language Version 1.1. the BPEL4WS Specification." 2003.

VANHATALO, J.;J. KOEHLER;F. LEYMANN. Repository for business processes and arbitrary associated metadata. BPM Demo Session at the Fourth International Conference on Business Process Management. Viena. Austria: 25-31, 2006.

W3C. (2001). "Wireless Application Protocol Forum, WAG UAProf "- Retrieved 7 de octubre de 2010, 2010, from http://www.wapforum.org/.

ZHENGHUI, W.;Q. XU TIANYIN;ZHUZHONG;L. SANGLU. A Parameter-Based Scheme for Service Composition in Pervasive Computing Environment. International Conference on Complex, Intelligent and Software Intensive Systems. Fukuoka, Japan: 543-548, 2009.

Recebido em maio de 2011

Aprovado para publicação em junho de 2011

Luis Javier Suarez Meza

Ingeniero en Electrónica y Telecomunicaciones de la Universidad del Cauca (Unicauca),Popayán - Colombia. Estudiante de Maestría en Ingeniería Telemática de la Unicauca e Investigador del Grupo de Ingeniería Telemática (GIT) de la Unicauca. Email: luisjavier.suarezmeza@gmail.com

Luis Antonio Rojas Potosí

Ingeniero en Electrónica y Telecomunicaciones de la Universidad del Cauca (Unicauca),Popayán - Colombia. Estudiante de Maestría en Ingeniería Telemática de la Unicauca e Investigador del Grupo de Ingeniería Telemática (GIT) de la Unicauca. Email: luisrojasmainmail@gmail.com

Victor Alberto Hermida

Ingeniero en Electrónica y Telecomunicaciones de la Universidad del Cauca (Unicauca),Popayán - Colombia. Magister en Ingeniería Telemática de la Unicauca. E-mail: luisrojasmainmail@gmail.com

Oscar Mauricio Caicedo

Magister en Ingeniería Telemática de la Unicauca. Estudiante de Doctorado de la Universidad Federal del Rio Grande del Sul (UFRGS), Porto Alegre-RS - Brasil. E-mail: omcaicedo@gmail.com

Juan Carlos Corrales

Doctor en Informática-Universidad de Versailles, Versailles - Francia. Docente de la Facultad de Ingeniería Electrónica y Telecomunicaciones (FIET) de la Unicauca, Popayán - Colombia. Coordinador del Grupo de Ingeniería Telemática (GIT). E-mail: juanco29@gmail.com 